\title{
The role of continuous subcutaneous insulin infusion therapy in a case of Seip-Berardinelli congenital lipodystrophy
}

\author{
Pedro Marques', Raquel Carvalho², Daniel Macedo', Sílvia Guerra² \\ 1Department of Endocrinology, Instituto Português de Oncologia de Lisboa, Portugal \\ 2Department of Endocrinology, Hospital Santa Maria, Lisboa, Portugal
}

E-mail: pedro.miguel.sousa.marques@gmail.com

\section{Introduction}

- Seip-Berardinelli congenital lipodystrophy (SBCL) is an extremely rare autosomal recessive disorder characterized by a congenital absence of adipose tissue.

- Hepatic steatosis, splenomegaly, skeletal muscle hypertrophy, hypertrophic cardiomyopathy, insulin-resistance and diabetes mellitus (DM) are some of the features of such patients.

\section{Case report}

- A caucasian-male patient, currently with 21 years-old, was referred at 6-months-age because of "muscular phenotype" and liver function alterations. On examination, muscular appearance with minimal subcutaneous fat (Figure 1) and hepatosplenomegaly were identified. Laboratorial tests revealed abnormalities in cholestatic liver enzymes; glycemia was normal. Ultrasound exams documented diffuse liver steatosis and hypertrophic cardiomyopathy. The identification of heterozygous BSCL2 gene mutation (p.Pro65ArgfsX28 and p.Thr109AsnfsX5) established the diagnosis of SBCL type 2.

- At 7 years old it was diagnosed DM (fasting plasma glucose= $182 \mathrm{mg} / \mathrm{dL} ; \mathrm{HbA} 1 \mathrm{C}=8.8 \%$; insulin=116.7mU/L; C-peptide=8.7 ng/mL; HOMA-IR=52.4). He was initially treated with metformin and later at 11 years old with concomitant insulin.

- He had always a poor metabolic control (Figure 2) justifying, at 16years old age the start on continuous subcutaneous insulin infusion (CSII) therapy and a significant improvement was obtained (last $\mathrm{HbA} 1 \mathrm{C}=8.0 \%)$. He is currently under metformin (4 g/day) and CSII therapy (daily dose of insulin of $155 \mathrm{U}$ ).

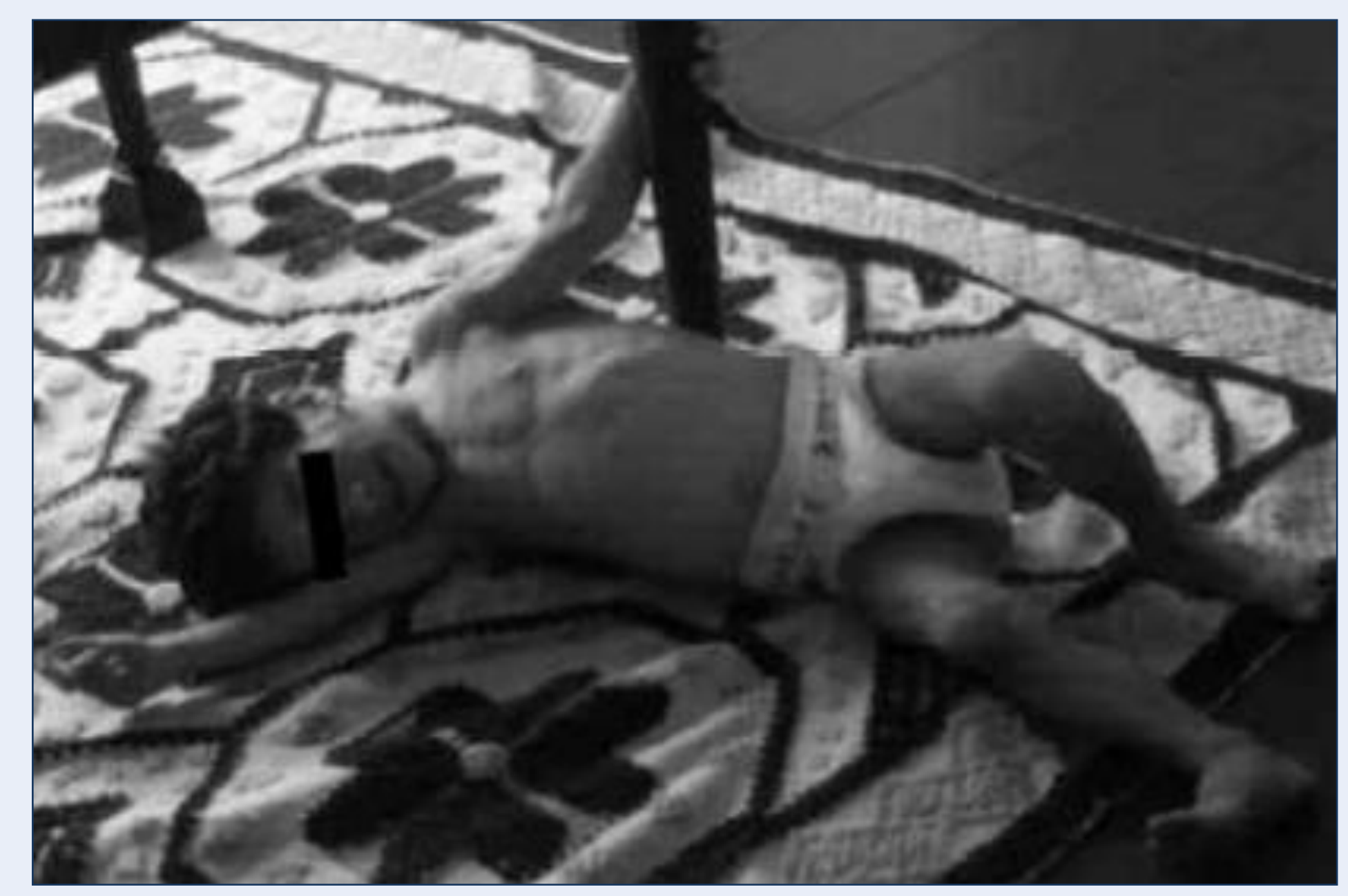

Figure 1: Old picture of the patient with the age of 11 months

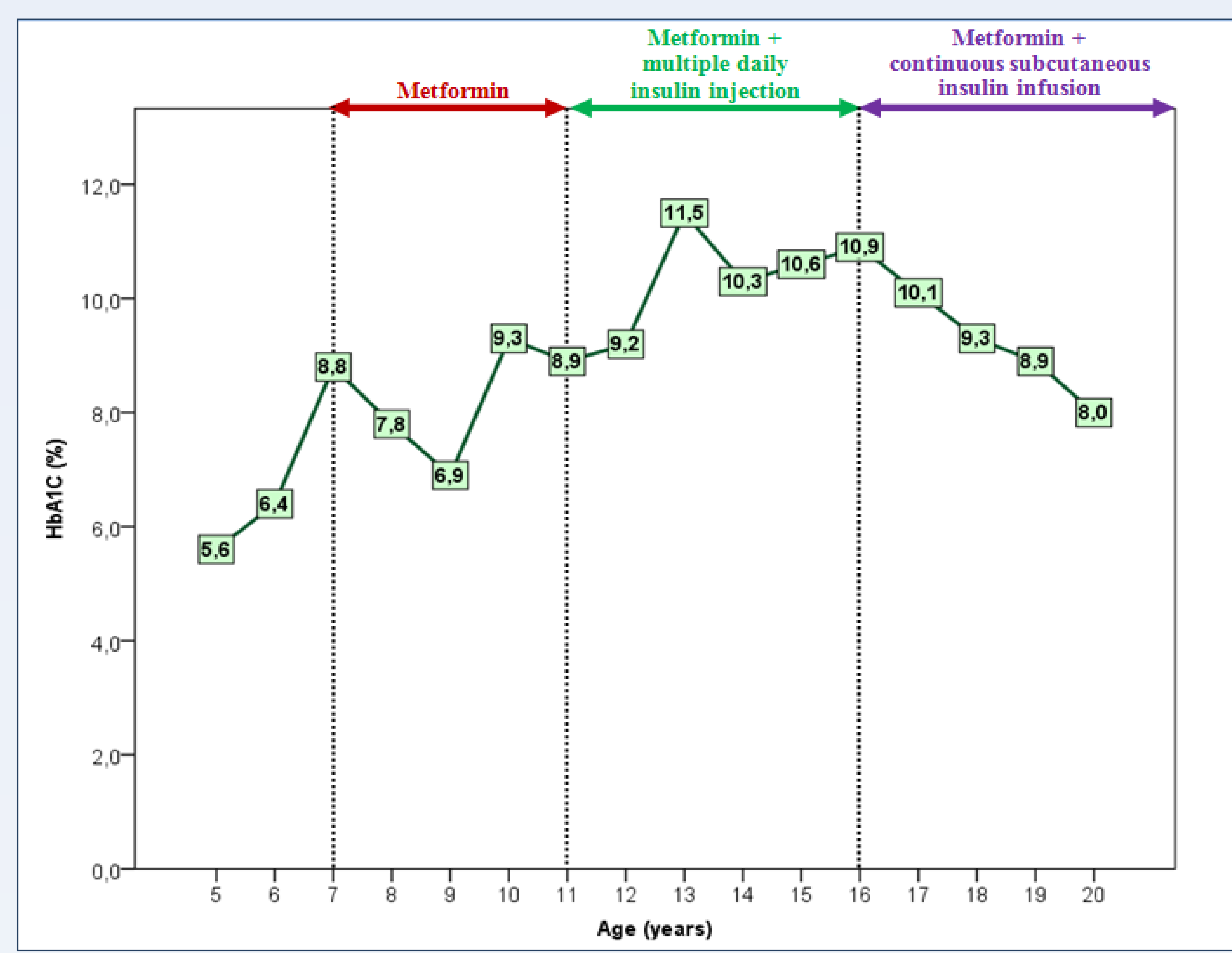

Figure 2: Evolution of $\mathrm{HbA1C}$ levels during the disease course Therapeutic approaches are indicated at the top of the figure.

\section{Discussion}

$>$ We report a rare case of lipoatrophic DM in childhood associated with severe insulin-resistance within the context of SBCL. In this setting, DM is frequently difficult to control and the management may involve insulin-sensitizers and exogenous insulin.

$>$ Although the CSII therapy is normally used in insulin-deficient patients, classically type $1 \mathrm{DM}$, some insulin-resistant DM patients, as the one reported, may benefit from this therapeutical approach. 